\title{
GENERALIZED FRIEZES AND A MODIFIED CALDERO-CHAPOTON MAP DEPENDING ON A RIGID OBJECT
}

\author{
THORSTEN HOLM AND PETER JØRGENSEN
}

\begin{abstract}
The (usual) Caldero-Chapoton map is a map from the set of objects of a category to a Laurent polynomial ring over the integers. In the case of a cluster category, it maps reachable indecomposable objects to the corresponding cluster variables in a cluster algebra. This formalizes the idea that the cluster category is a categorification of the cluster algebra. The definition of the Caldero-Chapoton map requires the category to be 2-Calabi-Yau, and the map depends on a cluster-tilting object in the category. We study a modified version of the Caldero-Chapoton map which requires only that the category have a Serre functor and depends only on a rigid object in the category. It is well known that the usual Caldero-Chapoton map gives rise to so-called friezes, for instance, Conway-Coxeter friezes. We show that the modified Caldero-Chapoton map gives rise to what we call generalized friezes and that, for cluster categories of Dynkin type $A$, it recovers the generalized friezes introduced by combinatorial means in recent work by the authors and Bessenrodt.
\end{abstract}

\section{$\S 0$. Introduction}

The (usual) Caldero-Chapoton map is an important object in the homological part of cluster theory (see [9, Section 3.1]). Among other things, it gives rise to so-called friezes. In particular, Conway-Coxeter friezes can be recovered like this (see [9, Section 5]).

This paper studies a modified version of the Caldero-Chapoton map. We show that it gives rise to what we call generalized friezes. In particular, the generalized friezes which were introduced by combinatorial means in [6] can be recovered like this.

Received November 7, 2013. Revised June 4, 2014. Accepted June 5, 2014.

2010 Mathematics Subject Classification. Primary 05E10; Secondary 13F60, 16G70, $18 \mathrm{E} 30$. 


\subsection{Background}

We first explain what the usual Caldero-Chapoton map is. If $Q$ is a finite quiver without loops and 2-cycles, then there is a cluster algebra $A(Q)$ and a cluster category $\mathrm{C}(Q)$ of type $Q$ (see [8], [16]).

The algebra $A(Q)$ and the category $\mathrm{C}(Q)$ are linked by the CalderoChapoton map $\rho_{T}$, which depends on a cluster-tilting object $T \in \mathrm{C}(Q)$ (see [9], [11], [12], [21], [22]). It is a map from the set of objects of $\mathrm{C}(Q)$ to a Laurent polynomial ring over $\mathbb{Z}$. Its image generates $A(Q)$, which embeds into Laurent polynomials. Indeed, $\rho_{T}$ maps reachable indecomposable objects to cluster variables and formalizes the idea that the cluster category is a categorification of the cluster algebra.

Note that $\rho_{T}$ can actually be defined on any 2-Calabi-Yau category C with a cluster-tilting object $T$, and that one of its good properties is that it is a so-called frieze (see [1, Definition 1.1], [9, Proposition 3.10], [15, Theorem]). This means that it is a map from the set of objects of $C$ to a ring, satisfying $\rho_{T}\left(c_{1} \oplus c_{2}\right)=\rho_{T}\left(c_{1}\right) \rho_{T}\left(c_{2}\right)$, such that if $\tau c \rightarrow b \rightarrow c$ is an Auslander-Reiten (AR) triangle in $C$, then

$$
\rho_{T}(\tau c) \rho_{T}(c)-\rho_{T}(b)=1
$$

Moreover, since $\rho_{T}$ has values in a Laurent polynomial ring over $\mathbb{Z}$, setting all the variables equal to 1 gives a frieze with values in $\mathbb{Z}$.

A classic case of this arises for $\mathrm{C}\left(A_{n}\right)$, the cluster category of Dynkin type $A_{n}$. For example, the $\mathrm{AR}$ quiver of $\mathrm{C}\left(A_{7}\right)$ is shown in Figure 1 . The quiver is $\mathbb{Z} A_{7}$ modulo a glide reflection, so the two dotted line segments in the figure should be identified with opposite orientations. Figure 2 shows a $\mathbb{Z}$-valued frieze, obtained as described, by giving its values on the indecomposable objects of $\mathrm{C}\left(A_{7}\right)$. Observe that (0.1) implies that if

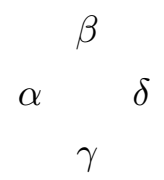

is a diamond in the frieze, then $\alpha \delta-\beta \gamma=1$. This is because such a diamond corresponds to a mesh in the AR quiver, hence to an AR triangle.

Friezes like this are known as Conway-Coxeter friezes and were studied long before cluster theory (see [13], [14]). They can also be defined by combinatorial means based on triangulations of polygons (see [7]). 


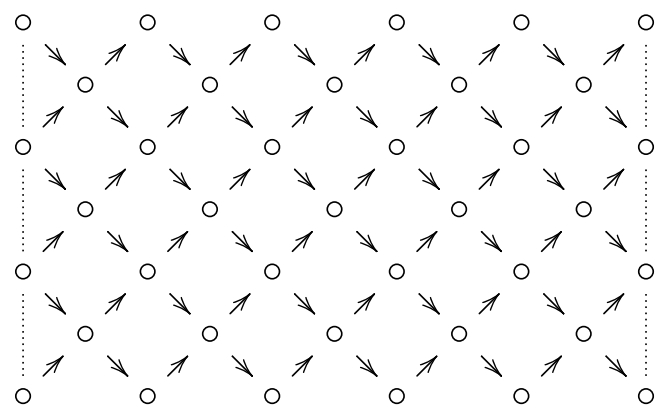

Figure 1: The Auslander-Reiten quiver of the cluster category $\mathrm{C}\left(A_{7}\right)$.

$\begin{array}{ccccccccccc}4 & & 4 & & 1 & & 2 & & 2 & & 4 \\ 11 & & 11 & & 2 & & 1 & & 10 & & 5 \\ & 15 & & 3 & & 1 & & 3 & & 7 & \vdots \\ 5 & 8 & & 7 & & 1 & & 3 & & 7 & \vdots \\ 5 & & 5 & & 3 & & 2 & & 2 & & 11 \\ & 3 & & 2 & & 5 & & 1 & & 3 & \vdots \\ 4 & & 1 & & 3 & & 2 & & 1 & & 4\end{array}$

Figure 2: A frieze on the cluster category $\mathrm{C}\left(A_{7}\right)$. This is also known as a Conway-Coxeter frieze.

\subsection{This paper}

We study a modified version of the Caldero-Chapoton map which does not require the category $\mathrm{C}$ to be 2 -Calabi-Yau but merely that it has a Serre functor. Moreover, it does not depend on a cluster-tilting object $T$ but on a rigid object $R$, that is, an object satisfying the weaker condition $\mathrm{C}(R, \Sigma R)=0$. Note that $\mathrm{C}(-,-)$ is shorthand for the Hom functor in $\mathrm{C}$.

To be precise, let $\mathbb{C}$ be the field of complex numbers, let $C$ be an essentially small $\mathbb{C}$-linear Hom-finite triangulated category with split idempotents and a Serre functor, let $R \in \mathrm{C}$ be a rigid object, and let $E=\mathrm{C}(R, R)$ be the 
endomorphism algebra. Consider the category $\operatorname{Mod} E$ of $E$-right modules and the functor

$$
\begin{aligned}
& \mathrm{C} \stackrel{G}{\longrightarrow} \operatorname{Mod} E, \\
& c \longmapsto \mathrm{C}(R, \Sigma c) .
\end{aligned}
$$

Note that $G$ actually has values in $\bmod E$, the category of $E$-modules which are finite-dimensional over $\mathbb{C}$, but we prefer to view it as having values in $\operatorname{Mod} E$ because of a later generalization.

The modified Caldero-Chapoton map determined by $R$ is given by the formula

$$
\rho_{R}(c)=\sum_{e} \chi\left(\operatorname{Gr}_{e}(G c)\right),
$$

where $c \in \mathrm{C}$ is an object, $\operatorname{Gr}_{e}(G c)$ is the Grassmannian of $E$-submodules $M \subseteq G c$ with $\mathrm{K}_{0}$-class satisfying $[M]=e$, and $\chi$ is the Euler characteristic defined by cohomology with compact support (see [17, p. 93]). The sum is over $e \in \mathrm{K}_{0}(\bmod E)$. This gives a map $\rho_{R}: \operatorname{obj} C \rightarrow \mathbb{Z}$.

One of our main results is the following.

TheOrem A. The map $\rho_{R}: \operatorname{objC} \rightarrow \mathbb{Z}$ is a generalized frieze. That is, (i) $\rho_{R}\left(c_{1} \oplus c_{2}\right)=\rho_{R}\left(c_{1}\right) \rho_{R}\left(c_{2}\right)$;

(ii) if $\Delta=\tau c \rightarrow b \rightarrow c$ is an $A R$ triangle in $\mathrm{C}$, then the difference $\rho_{R}(\tau c) \times$ $\rho_{R}(c)-\rho_{R}(b)$ equals 0 or 1 .

In fact, the difference in (ii) is 0 or 1 depending on whether $G(\Delta)$ is a split short exact sequence or not. If the difference in (ii) were always 1 , then $\rho_{R}$ would be a frieze in the earlier sense.

The idea of permitting the difference to be 0 or 1 occurred in [6], where generalized friezes on $\mathrm{C}\left(A_{n}\right)$ were introduced by purely combinatorial means based on higher angulations of polygons (see Paragraph 5.3 below for details). For example, Figure 3 shows the values of such a generalized frieze on the indecomposable objects of $\mathrm{C}\left(A_{7}\right)$. Note that for each diamond as in (0.2) we have $\alpha \delta-\beta \gamma$ equal to 0 or 1 .

It is another main result that the generalized friezes of [6] can be recovered from the modified Caldero-Chapoton map.

TheOrem B. Let $\mathrm{C}=\mathrm{C}\left(A_{n}\right)$ be the cluster category of type $A_{n}$. It follows from [10] that a rigid object $R \in \mathrm{C}$ without repeated indecomposable summands corresponds to a polygon dissection of an $(n+3)$-gon P. By [6] 


\begin{tabular}{|c|c|c|c|c|c|c|c|c|}
\hline & 2 & & 1 & & 1 & & 2 & \\
\hline 6 & & 2 & & 1 & & 2 & & 4 \\
\hline & 6 & & 1 & & 1 & & 4 & \\
\hline 6 & & 3 & & 1 & & 2 & & 4 \\
\hline & 3 & & 2 & & 1 & & 2 & \\
\hline 2 & & 2 & & 2 & & 1 & & 3 \\
\hline & 1 & & 2 & & 1 & & 1 & \\
\hline
\end{tabular}

Figure 3: A generalized frieze on the cluster category $\mathrm{C}\left(A_{7}\right)$, as introduced in [6].

such a polygon dissection defines a generalized frieze on $\mathrm{C}$, and this generalized frieze equals $\rho_{R}$.

Note that it is not explicit in [6] that its generalized friezes are defined on $\mathrm{C}\left(A_{n}\right)$, but it is established that they have the requisite periodicity to be so. Moreover, [6] requires that $R$ corresponds not just to a polygon dissection of $P$ but to a higher angulation. However, this turns out to be an unnecessary restriction, both for the combinatorial definition in [6] and for $\rho_{R}$.

This paper considers only the above version of the Caldero-Chapoton map with values in $\mathbb{Z}$. In the sequel [18] we consider a more elaborate version,

$$
\rho_{R}(c)=\alpha(c) \sum_{e} \chi\left(\operatorname{Gr}_{e}(G c)\right) \beta(e)
$$

where $\alpha$ and $\beta$ have values in a Laurent polynomial ring. In particular, we will obtain a version of the generalized friezes of [6] with values in Laurent polynomials.

The paper is organized as follows. Section 1 gives some background from representation theory, and Section 2 shows a few properties of Grassmannians. Section 3 proves Theorem A, Section 4 proves another useful property of $\rho_{R}$, and Section 5 proves Theorem B.

Note that Sections 1 and 2 sum up and adapt some well-known material to our setting. In these sections we make no claim to originality. However, it did not seem feasible to replace them with references. 


\section{§1. Modules over $\mathrm{R}$}

This section sums up some items from representation theory. Most of them go back to [2]-[4], and [5].

Setup 1.1. Throughout, $\mathbb{C}$ is the field of complex numbers and $\mathbb{C}$ is an essentially small $\mathbb{C}$-linear Hom-finite triangulated category with split idempotents and Serre functor $S$. The suspension functor of $\mathrm{C}$ is denoted $\Sigma$.

Moreover, $\mathrm{R}$ is a functorially finite subcategory of $\mathrm{C}$, closed under direct sums and summands, which is rigid; that is, $C(R, \Sigma R)=0$. Here $C(-,-)$ is short for $\operatorname{Hom}_{\mathrm{C}}(-,-)$.

1.2 (The case $\mathrm{R}=\operatorname{add} R$ ). An important special case is $\mathrm{R}=\operatorname{add} R$ where $R \in \mathrm{C}$ is rigid; that is, $\mathrm{C}(R, \Sigma R)=0$. Then $\mathrm{R}$ is automatically functorially finite, and we have the endomorphism algebra $E=\mathrm{C}(R, R)$, the category of $E$-right modules $\operatorname{Mod} E$, and the functor $G$ from (0.3). This is the situation from the Introduction.

However, $\mathrm{R}$ has the form add $R$ only when it has finitely many indecomposable objects, and we want to permit infinitely many because there are nice examples where it is relevant (see, e.g., [20, Section 6]). This requires the following, more general machinery.

1.3 (Krull-Schmidt categories). Since C is $\mathbb{C}$-linear Hom-finite with split idempotents, it is Krull-Schmidt. So is $\mathrm{R}$, since it is closed under direct sums and summands. We denote the sets of indecomposable objects by ind $C$ and ind $R$. Note that $R$ being rigid implies that $\Sigma^{-1}$ (ind $R$ ) and ind $R$ are disjoint.

1.4 (The category Mod R). We let Mod R $=\left(R^{o p}, \operatorname{Mod} \mathbb{C}\right)$ denote the category of $\mathbb{C}$-linear contravariant functors $\mathrm{R} \rightarrow \operatorname{Mod} \mathbb{C}$. It is an abelian category where a sequence $K \rightarrow L \rightarrow M$ is exact if and only if its evaluation at each object of $\mathrm{R}$ is exact (see [3, Section 2]).

There is a functor

$$
\begin{aligned}
& \mathrm{C} \stackrel{G}{\longrightarrow} \operatorname{Mod} \mathrm{R}, \\
& \left.c \longmapsto \mathrm{C}(-, \Sigma c)\right|_{\mathrm{R}} .
\end{aligned}
$$

Note that $G(\mathrm{R})=0$.

If $\mathrm{R}=\operatorname{add} R$ where $R$ is a rigid object, and $E=\mathrm{C}(R, R)$ is the endomorphism algebra, then there is an equivalence 


$$
\begin{aligned}
\operatorname{Mod} \mathrm{R} & \stackrel{\sim}{\longrightarrow} \operatorname{Mod} E, \\
M & \longmapsto M(R)
\end{aligned}
$$

which identifies the two versions of $G$ given in this paragraph and (0.3).

Note that $\operatorname{Mod} R=\left(R^{\text {op }}, \operatorname{Mod} \mathbb{C}\right)$ has the subcategory $\left(R^{\text {op }}, \bmod \mathbb{C}\right)$ of $\mathbb{C}$ linear contravariant functors $\mathrm{R} \rightarrow \bmod \mathbb{C}$. It is closed under subobjects and quotients, so it is an abelian subcategory of ModR with exact inclusion functor.

1.5 (Projective objects). An object $r \in \mathrm{R}$ gives a projective object

$$
P_{r}(-)=\mathrm{R}(-, r)=G\left(\Sigma^{-1} r\right)
$$

in Mod R. For an object $M \in$ ModR, Yoneda's lemma says that there is an isomorphism

$$
\operatorname{Hom}_{\operatorname{ModR}}\left(P_{r}, M\right) \rightarrow M(r)
$$

given by mapping a natural transformation $P_{r}=\mathrm{R}(-, r) \rightarrow M$ to its evaluation on $\mathrm{id}_{r}$.

If $r \in$ ind $\mathrm{R}$, then $P_{r}$ is indecomposable and has a unique maximal proper subobject, $\operatorname{rad} P_{r}$. Hence, a morphism $M \rightarrow P_{r}$ which is not an epimorphism factors through $\operatorname{rad} P_{r} \hookrightarrow P_{r}$ (see [3, Section 2], [4, Propositions 2.2 and 2.3]).

1.6 (The category $\bmod \mathrm{R}$ ). An object $M \in \operatorname{Mod} \mathrm{R}$ is called coherent if there is an exact sequence

$$
P_{r_{1}} \rightarrow P_{r_{0}} \rightarrow M \rightarrow 0
$$

with $r_{0}, r_{1} \in \mathrm{R}$. The full subcategory of coherent objects is denoted by mod R. It is clearly contained in $\left(R^{\text {op }}, \bmod \mathbb{C}\right)$. Since $R$ is functorially finite in $C$, the category $\bmod R$ is abelian by [19, Remark after Definition 2.9], and the inclusion $\bmod R \hookrightarrow \operatorname{Mod} R$ is exact by [2, Section III.2].

1.7 (Dualizing variety). Composition with the functor $\mathrm{D}(-)=\operatorname{Hom}_{\mathbb{C}}(-$, $\mathbb{C})$ gives a duality

$$
\left(\mathrm{R}^{\mathrm{op}}, \bmod \mathbb{C}\right) \rightarrow(\mathrm{R}, \bmod \mathbb{C}) .
$$

By [19, Propositions 2.10 and 2.11] the category $R$ is a dualizing variety in the sense of [5, Section 2], so the displayed duality restricts to a duality

$$
\bmod R \rightarrow \bmod R^{\text {op }}
$$


1.8 (Simple and finite length objects). The simple objects of ModR are precisely those of the form

$$
S_{r}=P_{r} / \operatorname{rad} P_{r}
$$

for $r \in$ ind $\mathrm{R}$ (see [4, Propositions 2.2 and 2.3]). Since $\mathrm{R}$ is a dualizing variety, $S_{r} \in \bmod \mathrm{R}$ for each $r \in$ ind R by [5, Proposition 3.2(c)]. As in [20, (1.4)] it follows that modR and Mod R have the same simple and the same finite length objects. We denote the full subcategory of finite length objects by $\mathrm{flR}$. It is closed under subobjects and quotients in $\bmod R$ and in Mod R, so it is abelian, and the inclusion functors $\mathrm{fl} \mathrm{R} \hookrightarrow \bmod \mathrm{R}$ and $\mathrm{fl} \mathrm{R} \hookrightarrow \mathrm{Mod} \mathrm{R}$ are exact.

1.9 (K-theory). It is immediate from Paragraph 1.8 that $\mathrm{K}_{0}(\mathrm{flR})$ is a free group on the generators $\left[S_{r}\right]$ for $r \in$ ind $\mathrm{R}$, where $[-]$ denotes the $\mathrm{K}_{0}$-class of an object. If $M \in \mathrm{flR}$, then $M$ has a finite filtration with simple quotients and the $\mathrm{K}_{0}$-class $[M]$ is the sum of the $\mathrm{K}_{0}$-classes of the simple quotients. For $M^{\prime} \subseteq M$ this implies that

$$
\left[M^{\prime}\right]=[M] \Leftrightarrow M^{\prime}=M, \quad\left[M^{\prime}\right]=0 \Leftrightarrow M^{\prime}=0
$$

1.10 (Injective objects). The previous items are left/right symmetric, so if $r \in$ ind R, then $\bar{P}_{r}=\mathrm{R}(r,-)$ is indecomposable projective in Mod Rop and there is a short exact sequence

$$
0 \rightarrow \operatorname{rad} \bar{P}_{r} \rightarrow \bar{P}_{r} \rightarrow \bar{S}_{r} \rightarrow 0
$$

in ModR $\mathrm{R}^{\mathrm{op}}$ where $\bar{S}_{r}$ is simple in ModR $\mathrm{R}^{\mathrm{op}}$. The sequence is in $(\mathrm{R}, \bmod \mathbb{C})$, and dualizing it gives a short exact sequence

$$
0 \rightarrow S_{r} \rightarrow I_{r} \rightarrow \operatorname{corad} I_{r} \rightarrow 0
$$

where

$$
I_{r}=\mathrm{DR}(r,-)=\mathrm{R}(-, S r)
$$

is indecomposable injective in ModR. A morphism $I_{r} \rightarrow N$ which is not a monomorphism factors through $I_{r} \rightarrow \operatorname{corad} I_{r}$.

The next two lemmas follow by standard methods. We include short proofs for completeness. Note that if $A$ and $B$ are full subcategories of $C$, then $\mathrm{A} * \mathrm{~B}$ denotes the full subcategory of objects $x$ appearing in distinguished triangles $a \rightarrow x \rightarrow b$ with $a \in \mathrm{A}, b \in \mathrm{B}$. 
LEMMA 1.11.

(i) For $M \in \bmod \mathrm{R}$ there is $z \in\left(\Sigma^{-1} \mathrm{R}\right) * \mathrm{R}$ such that $G z \cong M$.

(ii) For $z \in\left(\Sigma^{-1} \mathrm{R}\right) * \mathrm{R}$ and $c \in \mathrm{C}$, the map

$$
\mathrm{C}(z, c) \stackrel{G(-)}{\longrightarrow} \operatorname{Hom}_{\operatorname{Mod} \mathrm{R}}(G z, G c)
$$

is surjective.

Proof. (i) For $M \in \bmod \mathrm{R}$ there is an exact sequence $P_{r_{1}} \rightarrow P_{r_{0}} \rightarrow M \rightarrow 0$ with $r_{0}, r_{1} \in \mathrm{R}$. By (1.1) the first arrow is induced by a morphism $r_{1} \rightarrow r_{0}$ in R. Desuspending and completing to a distinguished triangle $\Sigma^{-1} r_{1} \rightarrow$ $\Sigma^{-1} r_{0} \rightarrow z \rightarrow r_{1}$ in $\mathrm{C}$, it is easy to check that $M \cong G z$.

(ii) For $r \in \mathrm{R},(1.1)$ gives an isomorphism $\operatorname{Hom}_{\operatorname{ModR}}\left(P_{r}, G c\right) \rightarrow(G c)(r)$, which can also be written $\operatorname{Hom}_{\operatorname{ModR}}\left(G\left(\Sigma^{-1} r\right), G c\right) \rightarrow \mathrm{C}\left(\Sigma^{-1} r, c\right)$. One checks that its inverse is $G(-)$, which is hence bijective in this case.

Now let $z \in\left(\Sigma^{-1} \mathrm{R}\right) * \mathrm{R}$ be given. There is a distinguished triangle $\Sigma^{-1} r_{1} \rightarrow$ $\Sigma^{-1} r_{0} \rightarrow z \rightarrow r_{1}$ which induces an exact sequence $G\left(\Sigma^{-1} r_{1}\right) \rightarrow G\left(\Sigma^{-1} r_{0}\right) \rightarrow$ $G z \rightarrow 0$ and a commutative diagram

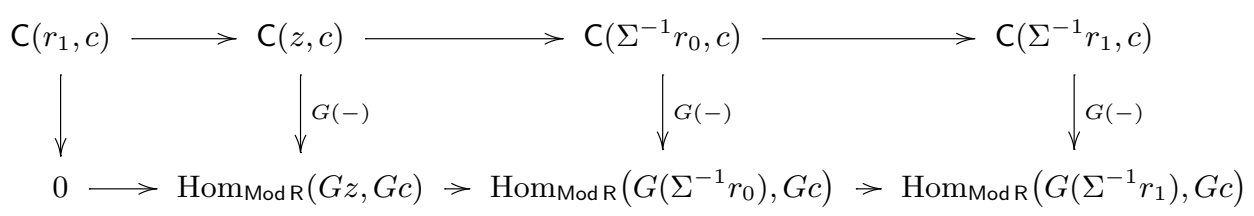

with exact rows. The first vertical arrow is surjective, and the third and fourth vertical arrows are bijective by the previous part of the proof. This four lemma implies that the second vertical arrow is surjective, as claimed.

Now let

$$
\Delta=\tau c \rightarrow b \stackrel{\beta}{\rightarrow} c
$$

be an AR triangle in $\mathrm{C}$, whence

$$
G(\Delta)=G(\tau c) \rightarrow G b \rightarrow G c
$$

is an exact sequence.

LEMMA 1.12 .

(i) If $c=\Sigma^{-1} r \in \Sigma^{-1}$ ind R, then $G(\Delta)=0 \rightarrow \operatorname{rad} P_{r} \rightarrow P_{r}$.

(ii) If $c=r \in$ ind R, then $G(\Delta)=I_{r} \rightarrow \operatorname{corad} I_{r} \rightarrow 0$.

(iii) If $c \notin \Sigma^{-1}$ (ind $\left.\mathrm{R}\right) \cup$ ind $\mathrm{R}$, then $G(\Delta)$ is a short exact sequence. 
Proof. (i) Let $c=\Sigma^{-1} r$, whence $G c=P_{r}$.

Pick a right R-approximation $r^{\prime} \stackrel{\rho^{\prime}}{\rightarrow} \Sigma b$. It is easy to see that composing with $\Sigma b \stackrel{\Sigma \beta}{\rightarrow} r$ gives a morphism $r^{\prime} \rightarrow r$ which is almost splitable in the sense of [4, Section 2], so the row in the following diagram is exact by [4, Corollary 2.6]:

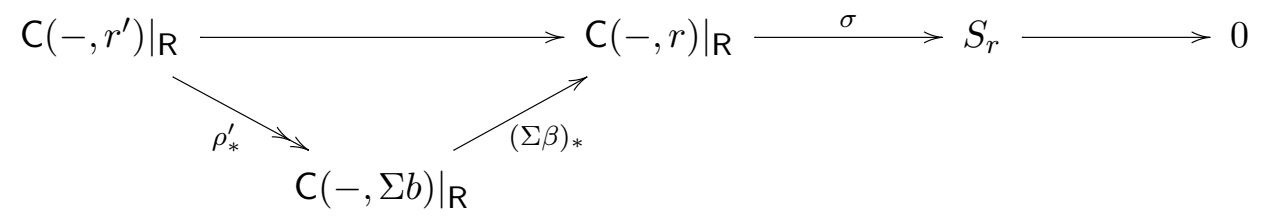

Since $\sigma$ is the canonical epimorphism $P_{r} \rightarrow S_{r}$, the diagram shows $\operatorname{Im}(\Sigma \beta)_{*}=\operatorname{rad} P_{r}$. This can also be written $\operatorname{Im} G \beta=\operatorname{rad} P_{r}$.

Finally, $c=\Sigma^{-1} r$ implies that

$$
\begin{aligned}
G(\tau c) & =\left.\mathrm{C}(-, \Sigma \tau c)\right|_{\mathrm{R}}=\left.\mathrm{C}\left(-, \Sigma\left(S \Sigma^{-1}\right)\left(\Sigma^{-1} r\right)\right)\right|_{\mathrm{R}} \\
& =\left.\mathrm{C}\left(-, S \Sigma^{-1} r\right)\right|_{\mathrm{R}}=\left.\mathrm{DC}\left(\Sigma^{-1} r,-\right)\right|_{\mathrm{R}}=0 .
\end{aligned}
$$

The sequence $G(\Delta)$ is exact and, combining with what we have shown, gives $G(\Delta)=0 \rightarrow \operatorname{rad} P_{r} \rightarrow P_{r}$ as desired.

(ii) Apply (i) to $C^{o p}$ and $R^{o p}$ and dualize.

(iii) There is a long exact sequence

$$
G\left(\Sigma^{-1} b\right) \stackrel{G\left(\Sigma^{-1} \beta\right)}{\longrightarrow} G\left(\Sigma^{-1} c\right) \longrightarrow G(\tau c) \longrightarrow G b \stackrel{G \beta}{\longrightarrow} G c .
$$

The first morphism can also be written $\left.\left.\mathrm{C}(-, b)\right|_{\mathrm{R}} \stackrel{\beta_{*}}{\rightarrow} \mathrm{C}(-, c)\right|_{\mathrm{R}}$. It is an epimorphism when $c \notin$ ind $\mathrm{R}$, since $\beta$ is right almost split. Similarly, the last morphism in the long exact sequence is an epimorphism when $\Sigma c \notin$ ind R, and (iii) of the proposition follows.

\section{§2. Grassmannians}

This section adapts some material from [9], [11], [12], [21], and [22] to our setting.

Definition 2.1 (Grassmannians). Let $M \in \operatorname{ModR}$ and $e \in \mathrm{K}_{0}(\mathrm{flR})$ be given. Let $\operatorname{Gr}(M)$ be the Grassmannian of subobjects $M^{\prime} \subseteq M$ with finite length, and let $\operatorname{Gr}_{e}(M) \subseteq \operatorname{Gr}(M)$ be the Grassmannian of subobjects $M^{\prime} \subseteq$ $M$ with finite length and $\left[M^{\prime}\right]=e$. 
2.2 (Constructible maps). A morphism $M \stackrel{j}{\rightarrow} N$ in $\mathrm{flR}$ induces constructible maps of Grassmannians as follows:

$$
\begin{aligned}
\operatorname{Gr}(M) & \rightarrow \operatorname{Gr}(N), & \operatorname{Gr}(N) & \rightarrow \operatorname{Gr}(M), \\
M^{\prime} & \mapsto j M^{\prime}, & N^{\prime} & \mapsto j^{-1} N^{\prime}
\end{aligned}
$$

(see [22, Section 2.1] for the definitions of constructible sets and maps). Note that, in particular, the image and the inverse image under a constructible map of a constructible set are constructible.

SETUP 2.3. For the rest of this section, $a \rightarrow b \rightarrow c$ are fixed morphisms in C. We assume that applying $G$ gives a short exact sequence

$$
0 \rightarrow G a \stackrel{i}{\rightarrow} G b \stackrel{p}{\rightarrow} G c \rightarrow 0
$$

and that $G a, G b, G c$ have finite length in Mod R.

Definition 2.4. For $e, f \in K_{0}(\mathrm{fl} \mathrm{R})$, there is a constructible subset

$$
X_{e, f}=\left\{L \in \operatorname{Gr}(G b) \mid\left[i^{-1} L\right]=e,[p L]=f\right\} \subseteq \operatorname{Gr}(G b)
$$

and a morphism

$$
\begin{aligned}
X_{e, f} & \stackrel{\pi_{e, f}}{\longrightarrow} \operatorname{Gr}_{e}(G a) \times \operatorname{Gr}_{f}(G c), \\
L \longmapsto & \left(i^{-1} L, p L\right) .
\end{aligned}
$$

Lemma 2.5. For each $g \in \mathrm{K}_{0}(\mathrm{fl} \mathrm{R})$ we have

$$
\operatorname{Gr}_{g}(G b)=\bigcup_{e+f=g} X_{e, f},
$$

where the right-hand side is a finite disjoint union.

Proof. Each $L \in \operatorname{Gr}(G b)$ is a subobject of $G b$ and so sits in a short exact sequence $0 \rightarrow i^{-1} L \rightarrow L \rightarrow p L \rightarrow 0$, whence $[L]=\left[i^{-1} L\right]+[p L]$ in $\mathrm{K}_{0}(\mathrm{flR})$. This gives the disjoint union in the lemma which is clearly finite.

LEMma 2.6.

(i) If the sequence (2.1) is split exact, then $\pi_{e, f}$ is surjective.

(ii) If $(e, f) \neq(0,[G c])$ and $a \rightarrow b \rightarrow c$ is an AR triangle, then $\pi_{e, f}$ is surjective. 
(iii) If $(e, f)=(0,[G c])$, then either $\pi_{e, f}$ is surjective or $X_{e, f}=\emptyset$. The former happens if and only if the sequence (2.1) is split exact.

(iv) If $(e, f)=(0,[G c])$, then $\operatorname{Gr}_{e}(G a) \times \operatorname{Gr}_{f}(G c)=\{(0, G c)\}$ has only one point.

(v) Each fiber of $\pi_{e, f}$ is an affine space over $\mathbb{C}$.

Proof. For (i) and (ii) let $(K, M) \in \mathrm{Gr}_{e}(G a) \times \mathrm{Gr}_{f}(G c)$ be given. That is, $K \subseteq G a, M \subseteq G c$ are subobjects with $[K]=e$ and $[M]=f$.

(i) When the sequence (2.1) is split exact, we set $L=K \oplus M \subseteq G a \oplus G c=$ $G b$, whence $i^{-1} L=K, p L=M$, so $\pi_{e, f}(L)=(K, M)$.

(ii) Pick $z \in\left(\Sigma^{-1} \mathrm{R}\right) * \mathrm{R}$ such that there is an isomorphism $G z \stackrel{\sim}{\rightarrow} M$ (see Lemma 1.11(i)). Composing it with the inclusion $M \subseteq G c$ gives a monomorphism $G z \rightarrow G c$ which has the form $G(z \stackrel{\zeta}{\rightarrow} c)$ by Lemma 1.11(ii). Note that $M=\operatorname{Im} G \zeta$.

First, suppose that $e \neq 0$. In this case, $K \neq 0$ by (1.2).

By Paragraphs 1.6 and 1.8 we can pick $r \in \mathrm{R}$ such that there is an epimorphism $P_{r}=G\left(\Sigma^{-1} r\right) \rightarrow K$. Composing it with the inclusion $K \subseteq G a$ gives a morphism $G\left(\Sigma^{-1} r\right) \rightarrow G a$ which has the form $G\left(\Sigma^{-1} r \stackrel{\varphi}{\rightarrow} a\right)$ by Lemma 1.11(ii). Note that $K=\operatorname{Im} G \varphi$ and that $K \neq 0$ implies that $\varphi \neq 0$.

We are assuming that there is an AR triangle $a \rightarrow b \rightarrow c \stackrel{\gamma}{\rightarrow} \Sigma a$, and since $\varphi$ and hence $\Sigma \varphi$ are nonzero, $\gamma$ factors as $c \stackrel{\varepsilon}{\rightarrow} r \stackrel{\Sigma \varphi}{\rightarrow} \Sigma a$. We can spin this into the following commutative diagram where the top row is also a distinguished triangle:

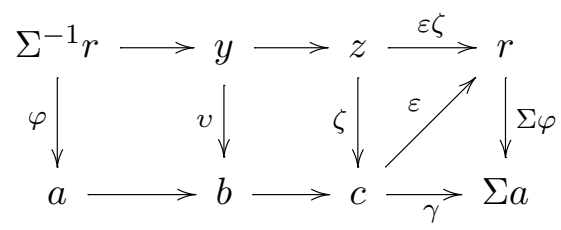

Applying $G$ gives a commutative diagram with exact rows:

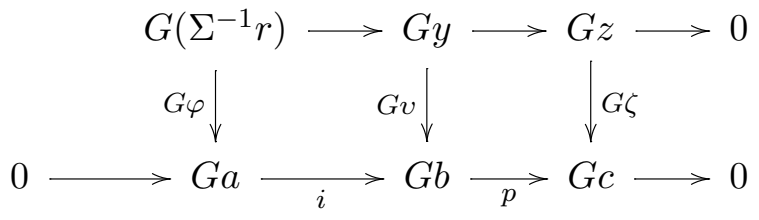

Set $L=\operatorname{Im} G v$. A diagram chase using that $G \zeta$ is a monomorphism shows that $i^{-1} L=\operatorname{Im} G \varphi=K$ and that $p L=\operatorname{Im} G \zeta=M$, so $\pi_{e, f}(L)=(K, M)$. 
Second, suppose that $e=0$. We are assuming that $(e, f) \neq(0,[G c])$, so $f \neq[G c]$ follows. In this case, $K=0$ and $M \neq G c$ by (1.2).

Since $M=\operatorname{Im} G \zeta$, the morphism $G z \stackrel{G \zeta}{\longrightarrow} G c$ is not an epimorphism; thus, $\zeta$ is not a split epimorphism, so $\zeta$ factors as $z \stackrel{v}{\rightarrow} b \rightarrow c$. Applying $G$ gives the following commutative diagram:



Set $L=\operatorname{Im} G v$. As above, a diagram chase using that $G \zeta$ is a monomorphism shows that $i^{-1} L=0=K$ and that $p L=\operatorname{Im} G \zeta=M$, so $\pi_{e, f}(L)=(K, M)$.

(iii) When $(e, f)=(0,[G c])$, it is clear from (iv) that either $\pi_{e, f}$ is surjective or $X_{e, f}=\emptyset$. The former happens if and only if there is a subobject $L \subseteq G b$ such that $i^{-1} L=0$ and $p L=G c$. This is clearly equivalent to the existence of a morphism $G c \stackrel{q}{\rightarrow} G b$ with $p q=\mathrm{id}$, that is, equivalent to the short exact sequence (2.1) being split exact.

(iv) This follows from (1.2).

(v) See [9, Lemma 3.11], which is stated for AR sequences but has a proof that also works in the present situation.

\section{$\S 3 . \quad$ Generalized friezes}

This section shows Theorem 3.3, which is a refined version of Theorem A from the Introduction.

Definition 3.1. For $c \in \mathrm{C}$ set

$$
\rho_{\mathrm{R}}(c)=\sum_{e} \chi\left(\operatorname{Gr}_{e}(G c)\right) .
$$

Recall that $G c$ is the R-module $\left.\mathrm{C}(-, \Sigma c)\right|_{\mathrm{R}}$ and that $\operatorname{Gr}_{e}(G c)$ is the Grassmannian of subobjects $M \subseteq G c$ with finite length and $[M]=e$, while $\chi$ is the Euler characteristic defined by cohomology with compact support (see $\left[17\right.$, p. 93]). The sum is over $e \in \mathrm{K}_{0}(\mathrm{flR})$.

Note that if $G c=0$, then $\rho_{\mathrm{R}}(c)=1$. However, for other objects $c$ the formula may not make sense because $G c$ may have infinite length, in which case the sum may be infinite. 
Definition 3.2. If $\mathrm{R}=\operatorname{add} R$ for a rigid object $R$, then we write $\rho_{R}$ instead of $\rho_{\mathrm{R}}$; this is the situation from the Introduction.

For the rest of this section and the next, $\rho_{\mathrm{R}}$ is abbreviated to $\rho$.

THEOREM 3.3.

(i) If $G c$ is of finite length, then the formula for $\rho(c)$ makes sense.

(ii) If $c_{1}, c_{2} \in \mathrm{C}$ have $G c_{1}, G c_{2}$ of finite length, then $G\left(c_{1} \oplus c_{2}\right)$ has finite length and $\rho\left(c_{1} \oplus c_{2}\right)=\rho\left(c_{1}\right) \rho\left(c_{2}\right)$.

(iii) If

$$
\Delta=\tau c \rightarrow b \rightarrow c
$$

is an $A R$ triangle in $\mathrm{C}$ and $G(\tau c)$, Gc have finite length, then so does $G b$ and

$$
\rho(\tau c) \rho(c)-\rho(b)= \begin{cases}0 & \text { if } G(\Delta) \text { is a split short exact sequence, } \\ 1 & \text { if } G(\Delta) \text { is not a split short exact sequence. }\end{cases}
$$

Proof. (i) If $G c$ has finite length, then $\operatorname{Gr}_{e}(G c)$ is nonempty only for finitely many values of $e$ (see [20, Paragraphs 1.6 and 1.8]). Hence, the formula for $\rho$ makes sense.

(iii) Consider the AR triangle $\Delta$, and suppose that $G(\tau c), G c$ have finite length. The exact sequence $G(\Delta)$ shows that $G b$ has finite length. We now split into cases.

Case (a): $c=\Sigma^{-1} r \in \Sigma^{-1}$ ind R. Lemma 1.12(i) says that

$$
G(\Delta)=0 \rightarrow \operatorname{rad} P_{r} \rightarrow P_{r}
$$

in particular, $G(\Delta)$ is not a split short exact sequence. We have

$$
\begin{aligned}
\rho(c) & =\sum_{e} \chi\left(\operatorname{Gr}_{e}\left(P_{r}\right)\right) \\
& =\chi\left(\operatorname{Gr}_{\left[P_{r}\right]}\left(P_{r}\right)\right)+\sum_{e \neq\left[P_{r}\right]} \chi\left(\operatorname{Gr}_{e}\left(P_{r}\right)\right) \\
& =1+\sum_{e} \chi\left(\operatorname{Gr}_{e}\left(\operatorname{rad} P_{r}\right)\right) \\
& =1+\rho(b) .
\end{aligned}
$$

The penultimate $=$ holds because (1.2) implies that $\operatorname{Gr}_{\left[P_{r}\right]}\left(P_{r}\right)=\left\{P_{r}\right\}$ has only one point and that each subobject $M \subseteq P_{r}$ with $[M] \neq\left[P_{r}\right]$ is proper and hence contained in $\operatorname{rad} P_{r}$. Moreover,

$$
\rho(\tau c)=1
$$


since $G(\tau c)=0$. Combining the equations shows that

$$
\rho(\tau c) \rho(c)-\rho(b)=1 .
$$

Case (b): $c=r \in$ ind R. We can use the dual argument to Case (a), based on Lemma 1.12(ii). We get that $G(\Delta)$ is not a split short exact sequence, and (3.1) remains true.

Case (c): $c \notin \Sigma^{-1}$ (ind R) $\cup$ ind R. We will use the machinery of Section 2, so set $a \rightarrow b \rightarrow c$ of Setup 2.3 equal to $\Delta=\tau c \rightarrow b \rightarrow c$. The requirements of the setup are satisfied because $G(\Delta)$ is a short exact sequence by Lemma 1.12(iii).

We have

$$
\begin{aligned}
\rho(\tau c) \rho(c)= & \sum_{e, f} \chi\left(\operatorname{Gr}_{e}(G(\tau c))\right) \chi\left(\operatorname{Gr}_{f}(G c)\right) \\
= & \sum_{e, f} \chi\left(\operatorname{Gr}_{e}(G(\tau c)) \times \operatorname{Gr}_{f}(G c)\right) \\
= & \chi\left(\operatorname{Gr}_{0}(G(\tau c)) \times \operatorname{Gr}_{[G c]}(G c)\right) \\
& +\sum_{(e, f) \neq(0,[G c])} \chi\left(\operatorname{Gr}_{e}(G(\tau c)) \times \operatorname{Gr}_{f}(G c)\right) \\
= & \chi\left(\operatorname{Gr}_{0}(G(\tau c)) \times \operatorname{Gr}_{[G c]}(G c)\right)+\sum_{(e, f) \neq(0,[G c])} \chi\left(X_{e, f}\right) .
\end{aligned}
$$

The second $=$ is by $[17$, p. 92 , item (4)], and the last $=$ is by $[17$, p. 93 , Exercise] and Lemma 2.6(ii), (v). On the other hand,

$$
\rho(b)=\sum_{g} \chi\left(\operatorname{Gr}_{g}(G b)\right)=\sum_{e, f} \chi\left(X_{e, f}\right)=\chi\left(X_{0,[G c]}\right)+\sum_{(e, f) \neq(0,[G c])} \chi\left(X_{e, f}\right),
$$

where the second $=$ is by $[17$, p. 92 , item (3)] and Lemma 2.5. It follows that

$$
\rho(\tau c) \rho(c)-\rho(b)=\chi\left(\operatorname{Gr}_{0}(G(\tau c)) \times \operatorname{Gr}_{[G c]}(G c)\right)-\chi\left(X_{0,[G c]}\right)=(\dagger) .
$$

If $G(\Delta)$ is split exact, then $\pi_{0,[G c]}$ is surjective by Lemma 2.6(i), whence $(\dagger)=0$ by $[17$, p. 93 , Exercise] and Lemma $2.6(\mathrm{v})$. If $G(\Delta)$ is not split exact, then Lemma 2.6(iii), (iv) implies that $(\dagger)=1-0=1$.

(ii) Suppose that $G c_{1}, G c_{2}$ have finite length. It is clear that $G\left(c_{1} \oplus c_{2}\right)$ has finite length. Set $a \rightarrow b \rightarrow c$ of Setup 2.3 equal to $c_{1} \rightarrow c_{1} \oplus c_{2} \rightarrow c_{2}$. 
A simplified version of the above computation for Case (c), using part (i) of Lemma 2.6 instead of (ii), shows that $\rho\left(c_{1} \oplus c_{2}\right)=\rho\left(c_{1}\right) \rho\left(c_{2}\right)$.

Definition 3.4. Let $A$ be a commutative ring. A generalized frieze on $\mathrm{C}$ with values in $A$ is a map $\varphi:$ objC $\rightarrow A$ satisfying

(i) $\varphi\left(c_{1} \oplus c_{2}\right)=\varphi\left(c_{1}\right) \varphi\left(c_{2}\right)$;

(ii) if $\tau c \rightarrow b \rightarrow c$ is an AR triangle in $\mathrm{C}$, then $\varphi(\tau c) \varphi(c)-\varphi(b)$ equals 0 or 1 .

Corollary 3.5. If Gc has finite length for each $c \in \mathrm{C}$, then $\rho$ is a generalized frieze with values in $\mathbb{Z}$.

Proof. This is immediate from Theorem 3.3.

REMARK 3.6. Theorem A in the Introduction follows from this since it is clear that each $G c$ has finite length when $\mathrm{R}=\operatorname{add} R$ for a rigid object $R$.

However, Theorem 3.3 is a bit finer because it also deals with situations where $\rho$ is not defined on every $c \in \mathrm{C}$.

\section{§4. An extension formula}

This section shows Proposition 4.4 which is akin to the exchange relation or multiplication property for cluster maps, albeit in a special case (see [12, Introduction] and [21, Introduction]).

SETuP 4.1. In this section $C$ is assumed to be 2-Calabi-Yau; that is, its Serre functor is $S=\Sigma^{2}$.

Moreover, $m \in$ ind $C$ and $r \in$ ind $\mathrm{R}$ denote objects satisfying

$$
\operatorname{dim}_{\mathbb{C}} \operatorname{Ext}_{\mathbb{C}}^{1}(r, m)=\operatorname{dim}_{\mathbb{C}} \operatorname{Ext}_{\mathbb{C}}^{1}(m, r)=1,
$$

and $m \rightarrow a \rightarrow r$ and $r \rightarrow b \rightarrow m$ are the ensuing nonsplit extensions.

REMARK 4.2. Being more verbose, we have the following distinguished triangles with $\delta, \varepsilon \neq 0$ :

$$
m \stackrel{\mu}{\rightarrow} a \rightarrow r \stackrel{\delta}{\rightarrow} \Sigma m, \quad r \rightarrow b \stackrel{\beta}{\rightarrow} m \stackrel{\varepsilon}{\rightarrow} \Sigma r .
$$

Applying $G$ gives exact sequences in Mod R:

$$
G\left(\Sigma^{-1} r\right) \stackrel{G\left(\Sigma^{-1} \delta\right)}{\longrightarrow} G m \stackrel{G \mu}{\longrightarrow} G a \rightarrow 0, \quad 0 \rightarrow G b \stackrel{G \beta}{\longrightarrow} G m \stackrel{G \varepsilon}{\longrightarrow} G(\Sigma r) .
$$

LEMmA 4.3. If $M \subseteq G m$, then either $\operatorname{Ker} G \mu \subseteq M$ or $M \subseteq \operatorname{Im} G \beta$, but not both. 
Proof. Equivalently, either $\operatorname{Im} G\left(\Sigma^{-1} \delta\right) \subseteq M$ or $M \subseteq \operatorname{Ker} G \varepsilon$, but not both.

Not both: Since C is 2-Calabi-Yau, its AR translation is $\tau=\Sigma$, so there is an AR triangle $\Sigma r \rightarrow y \rightarrow r \stackrel{\sigma}{\rightarrow} \Sigma^{2} r$. The morphism $r \stackrel{\delta}{\rightarrow} \Sigma m$ is nonzero, so $\sigma$ factors as $r \stackrel{\delta}{\rightarrow} \Sigma m \stackrel{\psi}{\rightarrow} \Sigma^{2} r$. Since $\psi \delta=\sigma \neq 0$, we have $\psi \neq 0$. It therefore follows from $\operatorname{dim}_{\mathbb{C}} \mathrm{C}\left(\Sigma m, \Sigma^{2} r\right)=\operatorname{dim}_{\mathbb{C}} \mathrm{C}(m, \Sigma r)=1$ that $\Sigma m \stackrel{\Sigma \varepsilon}{\longrightarrow} \Sigma^{2} r$ is a nonzero scalar multiple of $\psi$, whence $\psi \delta \neq 0$ implies that $\Sigma(\varepsilon) \delta \neq 0$. Hence, $G\left(\varepsilon \Sigma^{-1} \delta\right) \neq 0$, because this morphism is

$$
\left.\left.\mathrm{C}(-, r)\right|_{\mathrm{R}} \stackrel{(\Sigma(\varepsilon) \delta)_{*}}{\longrightarrow} \mathrm{C}\left(-, \Sigma^{2} r\right)\right|_{\mathrm{R}}
$$

Now suppose that $\operatorname{Im} G\left(\Sigma^{-1} \delta\right) \subseteq M$. Applying $G \varepsilon$ gives $\operatorname{Im} G\left(\varepsilon \Sigma^{-1} \delta\right) \subseteq$ $(G \varepsilon) M$. By what we have shown above, this implies that $(G \varepsilon) M \neq 0$, that is, that $M \nsubseteq \operatorname{Ker} G \varepsilon$ as claimed.

Either/or: Suppose that $M \nsubseteq \operatorname{Ker} G \varepsilon$. Since $G \varepsilon$ is

$$
\left.\left.\mathrm{C}(-, \Sigma m)\right|_{\mathrm{R}} \stackrel{(\Sigma \varepsilon)_{*}}{\longrightarrow} \mathrm{C}\left(-, \Sigma^{2} r\right)\right|_{\mathrm{R}}
$$

this means that there exist $r^{\prime} \in$ ind $\mathrm{R}$ and a morphism $r^{\prime} \stackrel{\rho^{\prime}}{\rightarrow} \Sigma m$ in $M\left(r^{\prime}\right)$ such that the composition $r^{\prime} \stackrel{\rho^{\prime}}{\rightarrow} \Sigma m \stackrel{\Sigma \varepsilon}{\rightarrow} \Sigma^{2} r$ is nonzero. Hence, the map $\mathrm{C}\left(\Sigma m, \Sigma^{2} r\right) \stackrel{\rho^{\prime *}}{\rightarrow} \mathrm{C}\left(r^{\prime}, \Sigma^{2} r\right)$ is nonzero, whence the lower horizontal map is nonzero in the following commutative square which exists by Serre duality:

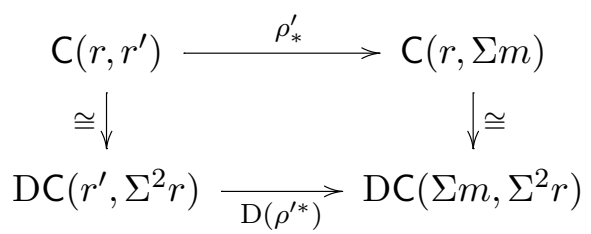

It follows that the upper horizontal map is nonzero and so is surjective since $\operatorname{dim}_{\mathbb{C}} \mathrm{C}(r, \Sigma m)=1$ by assumption. Hence, $r \stackrel{\delta}{\rightarrow} \Sigma m$ factors as $r \rightarrow r^{\prime} \stackrel{\rho^{\prime}}{\rightarrow} \Sigma m$.

However, for $r^{\prime \prime} \in \mathrm{R}$ each element of $\left(\operatorname{Im} G\left(\Sigma^{-1} \delta\right)\right)\left(r^{\prime \prime}\right)$ is a composition $r^{\prime \prime} \rightarrow r \stackrel{\delta}{\rightarrow} \Sigma m$. By what we have shown, such a composition can also be written as a composition $r^{\prime \prime} \rightarrow r^{\prime} \stackrel{\rho^{\prime}}{\rightarrow} \Sigma m$ and so is in $M\left(r^{\prime \prime}\right)$. Hence, $\operatorname{Im} G\left(\Sigma^{-1} \delta\right) \subseteq M$ as desired.

Proposition 4.4. In the situation of Setup 4.1, if $G m$ has finite length, then so do Ga and $G b$, and

$$
\rho(m)=\rho(a)+\rho(b) .
$$


Proof. The claim about lengths follows from the exact sequences in Remark 4.2.

When $G m$ has finite length there are injections

$$
\begin{aligned}
\operatorname{Gr}_{e-[\operatorname{Ker} G \mu]}(G a) \longleftrightarrow & \operatorname{Gr}_{e}(G m) \longleftrightarrow \operatorname{Gr}_{e}(G b), \\
K \longmapsto & (G \mu)^{-1} K, \\
(G \beta) L \longleftarrow & L .
\end{aligned}
$$

The images are constructible by Paragraph 2.2, and they are disjoint with union equal to $\operatorname{Gr}_{e}(G m)$ by Lemma 4.3, whence

$$
\chi\left(\operatorname{Gr}_{e}(G m)\right)=\chi\left(\operatorname{Gr}_{e-[\operatorname{Ker} G \mu]}(G a)\right)+\chi\left(\operatorname{Gr}_{e}(G b)\right)
$$

by $\left[17\right.$, p. 92, item (3)]. Summing over $e \in \mathrm{K}_{0}(\mathbf{f l R})$ proves the proposition.

Remark 4.5. Since $G r=0$, we have $\rho(r)=1$, so Proposition 4.4 can also be written as

$$
\rho(m) \rho(r)=\rho(a)+\rho(b)
$$

This makes it clearer that it is akin to the exchange relation or multiplication property for cluster characters (see [12, Introduction], [21, Introduction]).

If $r \in$ ind C, then (4.1) holds for cluster characters but may fail for $\rho$ (see Remark 5.5).

\section{$\S 5$. The generalized friezes of [6]}

This section shows Theorem 5.4, which is a reformulation of Theorem B in the Introduction.

SETup 5.1. In this section, $n \geq 3$ is an integer, $\mathrm{C}=\mathrm{C}\left(A_{n}\right)$ is the cluster category of type $A_{n}$ (see [8], [10]), and $R$ is a rigid object of $\mathrm{C}$ without repeated indecomposable summands. We set $\mathrm{R}=\operatorname{add} R$ (see Paragraph 1.2).

5.2 (Coordinates and diagonals). It is clear that $G c$ has finite length for each $c \in \mathrm{C}$, and it is well known that $\mathrm{C}$ and $\mathrm{R}$ satisfy the conditions of Setups 1.1 and 4.1, so the results of Sections 3 and 4 apply.

The following properties were shown in [10]. The AR quiver of $\mathrm{C}$ is $\mathbb{Z} A_{n}$ modulo a certain glide reflection. There is a coordinate system on the AR 


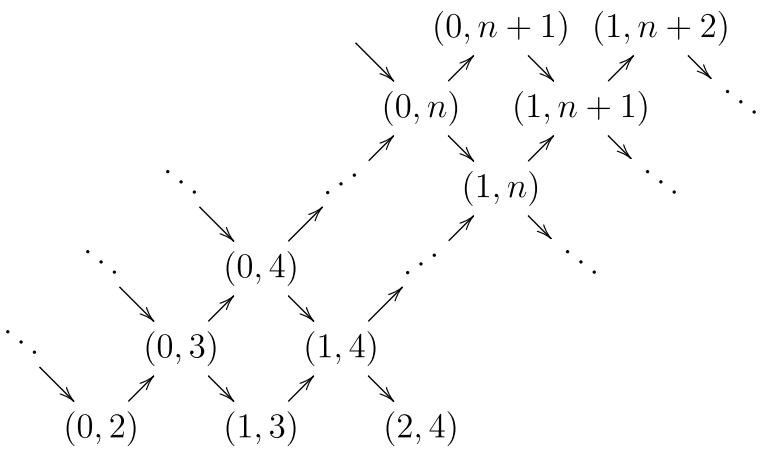

Figure 4: The coordinate system on the $\mathrm{AR}$ quiver of $\mathrm{C}\left(A_{n}\right)$.

quiver of C, part of which is shown in Figure 4. It is continued with the stipulations that the order of the coordinates does not matter and that individual coordinates are taken modulo $n+3$; this emulates the action of the glide reflection. We think of the coordinate pair $(i, j)$ as the diagonal connecting vertices $i$ and $j$ in a regular $(n+3)$-gon $P$ with vertex set $\{0, \ldots, n+2\}$. This identifies the indecomposable objects of $C$ with the diagonals of $P$. The identification has the property that if $M, S \in$ ind C, then

$$
\operatorname{dim}_{\mathbb{C}} \operatorname{Ext}_{\mathbb{C}}^{1}(M, S)= \begin{cases}1 & \text { if } M \text { and } S \text { cross } \\ 0 & \text { if not. }\end{cases}
$$

In particular, the indecomposable summands of the rigid object $R$ are a set of pairwise noncrossing diagonals of $P$, that is, a polygon dissection of $P$ which will also be denoted by $R$.

5.3 (The generalized friezes of [6]). Let us recall the algorithm of $[6$, Section 3], which uses the polygon dissection $R$ of the $(n+3)$-gon $P$ to define a generalized frieze on $\mathrm{C}=\mathrm{C}\left(A_{n}\right)$. Note that in [6] the polygon dissection was assumed to be a higher angulation, but this restriction is unnecessary.

Define nonnegative integers $m_{R}(i, j)$, indexed by vertices $i, j$ of $P$, by the following inductive procedure. Let $i$ be fixed. Set $m_{R}(i, i)=0$. The polygon dissection $R$ splits $P$ into smaller polygonal pieces. If $\alpha$ is a piece containing $i$, and $j$ is another vertex of $\alpha$, then set $m_{R}(i, j)=1$. If $\alpha$ is a piece not containing $i$, then we can assume that there is a piece $\alpha^{\prime}$ sharing an edge 
$(k, \ell)$ with $\alpha$, such that $m_{R}(i, j)$ has already been defined for the vertices $j$ of $\alpha^{\prime}$. Set

$$
m_{R}(i, j)=m_{R}(i, k)+m_{R}(i, \ell)
$$

for each vertex $j \neq k, \ell$ of $\alpha$. Note that $(k, \ell)$ is a diagonal in $R$, that is, an indecomposable summand of $R$.

It was proved in [6, Theorem 3.3] that $m_{R}(i, j)=m_{R}(j, i)$, so $m_{R}$ can be viewed as being defined on the diagonals of $P$, that is, on the indecomposable objects of $\mathrm{C}$. It is extended to all objects by the rule $m_{R}\left(c_{1} \oplus c_{2}\right)=$ $m_{R}\left(c_{1}\right) m_{R}\left(c_{2}\right)$.

Moreover, the AR triangles in $\mathrm{C}$ have the form

$$
(i-1, j-1) \rightarrow(i-1, j) \oplus(i, j-1) \rightarrow(i, j)
$$

where $(i-1, j)$ and $(i, j-1)$ have to be interpreted as 0 if their coordinates are neighboring vertices of $P$, and it was proved in [6, Theorem 5.1] that each difference

$$
m_{R}(i-1, j-1) m_{R}(i, j)-m_{R}(i-1, j) m_{R}(i, j-1)
$$

equals 0 or 1 .

Hence, $m_{R}$ is a generalized frieze on $\mathrm{C}$.

Theorem 5.4. Consider the situation of Setup 5.1. The rigid object $R$ gives a polygon dissection of the $(n+3)$-gon $P$ (see Paragraph 5.2), and the dissection gives a generalized frieze $m_{R}$ on $\mathrm{C}$ (see Paragraph 5.3).

The rigid object $R$ also gives a generalized frieze $\rho_{R}$ on C (see Definition 3.1 and Corollary 3.5).

These generalized friezes agree; that is, $m_{R}=\rho_{R}$.

Proof. Since $m_{R}\left(c_{1} \oplus c_{2}\right)=m_{R}\left(c_{1}\right) m_{R}\left(c_{2}\right)$ by definition and since $\rho_{R}\left(c_{1} \oplus\right.$ $\left.c_{2}\right)=\rho_{R}\left(c_{1}\right) \rho_{R}\left(c_{2}\right)$ by Theorem 3.3(ii), it is enough to let $i$ be a fixed vertex of $P$ and to show that

$$
m_{R}(i, j)=\rho_{R}((i, j))
$$

for each vertex $j$ of $P$, and we do so inductively. 
The polygon dissection $R$ splits $P$ into smaller polygonal pieces. If $\alpha$ is a piece containing $i$, and $j$ is another vertex of $\alpha$, then by definition $m_{R}(i, j)=1$. The diagonal $(i, j)$ crosses none of the diagonals in $R$, so $\operatorname{Ext}_{\mathrm{C}}^{1}(R,(i, j))=0$ by (5.1). That is, $G((i, j))=0$, so $\rho_{R}((i, j))=1$, verifying (5.4).

If $\alpha$ is a piece not containing $i$, then we can assume that there is a piece $\alpha^{\prime}$ sharing an edge $S=(k, \ell)$ with $\alpha$, such that, if $j$ is a vertex of $\alpha^{\prime}$, then (5.4) has already been verified, and such that, if $j \neq k, \ell$ is a vertex of $\alpha$, then $M=(i, j)$ crosses $S$. For such a $j$,

$$
\operatorname{dim}_{\mathbb{C}} \operatorname{Ext}_{\mathbb{C}}^{1}(M, S)=\operatorname{dim}_{\mathbb{C}} \operatorname{Ext}_{\mathbb{C}}^{1}(S, M)=1
$$

by (5.1), and there are nonsplit extensions

$$
M \rightarrow A \oplus A^{\prime} \rightarrow S, \quad S \rightarrow B \oplus B^{\prime} \rightarrow M
$$

in $\mathrm{C}$ where $A, A^{\prime}, B, B^{\prime} \in$ ind $\mathrm{C}$ are the diagonals in Figure 5 . Note that if one or more of $A, A^{\prime}, B, B^{\prime}$ are edges of $P$, then they must be interpreted as zero objects, and note that $S$ is a diagonal in $R$, that is, an indecomposable summand of $R$.

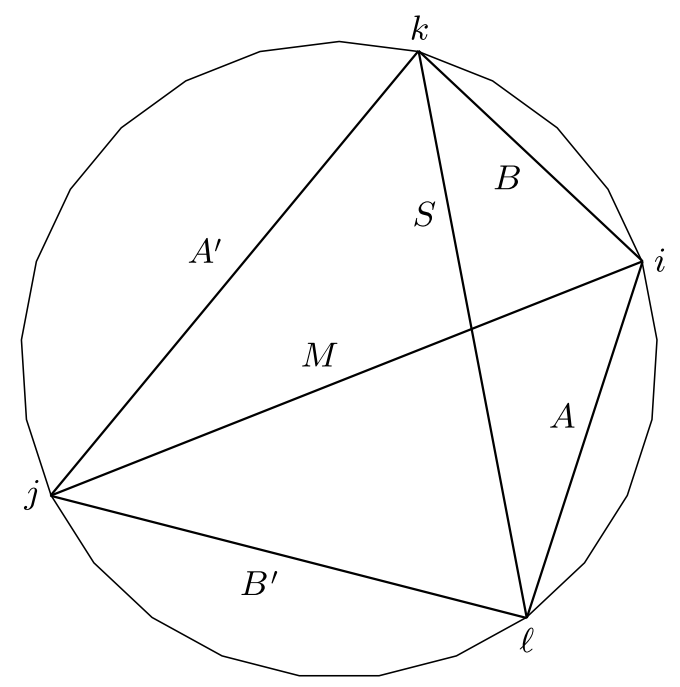

Figure 5: There are nonsplit extensions $M \rightarrow A \oplus A^{\prime} \rightarrow S$ and

$$
S \rightarrow B \oplus B^{\prime} \rightarrow M \text { in } \mathrm{C}\left(A_{n}\right) .
$$


Combining Proposition 4.4 and Theorem 3.3(ii) gives

$$
\rho_{R}(M)=\rho_{R}(A) \rho_{R}\left(A^{\prime}\right)+\rho_{R}(B) \rho_{R}\left(B^{\prime}\right) .
$$

Since $j, k, \ell$ are vertices of $\alpha$, the diagonals $A^{\prime}=(j, k)$ and $B^{\prime}=(j, \ell)$ cross none of the diagonals in $R$, so $G A^{\prime}=G B^{\prime}=0$ by (5.1), and hence $\rho_{R}\left(A^{\prime}\right)=$ $\rho_{R}\left(B^{\prime}\right)=1$. Equation (5.5) therefore reads $\rho_{R}(M)=\rho_{R}(A)+\rho_{R}(B)$, giving the first of the following equalities:

$$
\rho_{R}((i, j))=\rho_{R}((i, \ell))+\rho_{R}((i, k))=m_{R}(i, \ell)+m_{R}(i, k)=m_{R}(i, j) .
$$

The second equality is by assumption since $k, \ell$ are vertices of $\alpha^{\prime}$, and the third equality is (5.2).

This shows (5.4) for the vertices $j$ of $\alpha$, completing the induction.

REMARK 5.5. Consider the situation of Setup 4.1. Remark 4.5 proved (4.1) for $r \in$ ind R. The remark claimed that if $r \in$ ind C, then (4.1) may fail. We can now prove this: if it did always hold, then for $\mathrm{C}=\mathrm{C}\left(A_{n}\right)$ we could let the extensions in Setup 4.1 be

$$
(i-1, j-1) \rightarrow(i-1, j) \oplus(i, j-1) \rightarrow(i, j), \quad(i, j) \rightarrow 0 \rightarrow(i-1, j-1),
$$

where the first is the AR triangle ending in $(i, j)$ and the second has connecting morphism equal to the identity on $(i-1, j-1)$. Then (4.1) would give

$$
\rho_{R}((i-1, j-1)) \rho_{R}((i, j))=\rho_{R}((i-1, j)) \rho_{R}((i, j-1))+1,
$$

and Theorem 5.4 would imply that the difference (5.3) was always 1 . That is false, however (see [6, Theorem 5.1(c)]).

Acknowledgments. This article grew out of [6]. We are grateful to Christine Bessenrodt for the fruitful collaboration on that article. We are also grateful for very useful input from Yann Palu and Pierre-Guy Plamondon. In particular, Pierre-Guy Plamondon visited Newcastle in March 2014, and his comments to the second author led to significant improvements. We also thank the referee for a number of useful comments.

Part of this work was carried out while Peter Jørgensen was visiting Hannover. He thanks Christine Bessenrodt, Thorsten Holm, and the Institut für Algebra, Zahlentheorie und Diskrete Mathematik at the Leibniz Universität for their hospitality. He also gratefully acknowledges financial support from Thorsten Holm's grant HO 1880/5-1, which is part of the research priority program SPP 1388 Darstellungstheorie of the Deutsche Forschungsgemeinschaft. 


\section{REFERENCES}

[1] I. Assem and G. Dupont, Friezes and a construction of the Euclidean cluster variables, J. Pure Appl. Algebra 215 (2011), 2322-2340. MR 2793939. DOI 10.1016/j.jpaa.2010.12.013.

[2] M. Auslander, "Representation dimension of Artin algebras," reprint of the 1971 original, in Selected Works of Maurice Auslander, Vol. 1, Amer. Math. Soc., Providence, 1999, 505-574.

[3] — Representation theory of Artin algebras, I, Comm. Algebra 1 (1974), 177268. MR 0349747.

[4] - Representation theory of Artin algebras, II, Comm. Algebra 1 (1974), 269310. MR 0349747.

[5] M. Auslander and I. Reiten, Stable equivalence of dualizing R-varieties, Adv. Math. 12 (1974), 306-366. MR 0342505.

[6] C. Bessenrodt, T. Holm, and P. Jørgensen, Generalized frieze pattern determinants and higher angulations of polygons, J. Combin. Theory Ser. A 123 (2014), 30-42. MR 3157797. DOI 10.1016/j.jcta.2013.11.003.

[7] D. Broline, D. W. Crowe, and I. M. Isaacs, The geometry of frieze patterns, Geom. Dedicata 3 (1974), 171-176. MR 0363955.

[8] A. B. Buan, R. Marsh, M. Reineke, I. Reiten, and G. Todorov, Tilting theory and cluster combinatorics, Adv. Math. 204 (2006), 572-618. MR 2249625. DOI 10.1016/j.aim.2005.06.003.

[9] P. Caldero and F. Chapoton, Cluster algebras as Hall algebras of quiver representations, Comment. Math. Helv. 81 (2006), 595-616. MR 2250855. DOI $10.4171 / \mathrm{CMH} / 65$.

[10] P. Caldero, F. Chapoton, and R. Schiffler, Quivers with relations arising from clusters ( $A_{n}$ case), Trans. Amer. Math. Soc. 358 (2006), no. 3, 1347-1364. MR 2187656. DOI 10.1090/S0002-9947-05-03753-0.

[11] P. Caldero and B. Keller, From triangulated categories to cluster algebras, I, Invent. Math. 172 (2008), 169-211. MR 2385670. DOI 10.1007/s00222-008-0111-4.

[12] - From triangulated categories to cluster algebras, II, Ann. Sci. Éc. Norm. Supér. (4) 39 (2006), 983-1009. MR 2316979. DOI 10.1016/j.ansens.2006.09.003.

[13] J. H. Conway and H. S. M. Coxeter, Triangulated polygons and frieze patterns, Math. Gaz. 57 (1973), 87-94. MR 0461269.

[14] - Triangulated polygons and frieze patterns, Math. Gaz. 57 (1973), 175-183. MR 0461270.

[15] S. Domínguez and C. Geiss, A Caldero-Chapoton formula for generalized cluster categories, J. Algebra 399 (2014), 887-893. MR 3144617. DOI 10.1016/j.jalgebra.2013.10.018.

[16] S. Fomin and A. Zelevinsky, Cluster algebras, I: Foundations, J. Amer. Math. Soc. 15 (2002), 497-529. MR 1887642. DOI 10.1090/S0894-0347-01-00385-X.

[17] W. Fulton, Introduction to Toric Varieties, Ann. of Math. Stud. 131, Princeton University Press, Princeton, 1993. MR 1234037.

[18] T. Holm and P. Jørgensen, Generalised friezes and a modified Caldero-Chapoton map depending on a rigid object, II, preprint, arXiv:1401.4616v1 [math.RT]

[19] O. Iyama and Y. Yoshino, Mutation in triangulated categories and rigid Cohen-Macaulay modules, Invent. Math. 172 (2008), 117-168. MR 2385669. DOI 10.1007/s00222-007-0096-4. 
[20] P. Jørgensen and Y. Palu, A Caldero-Chapoton map for infinite clusters, Trans. Amer. Math. Soc. 365 (2013), no. 3, 1125-1147. MR 3003260. DOI 10.1090/S0002-9947-2012-05464-X.

[21] Y. Palu, Cluster characters for 2-Calabi-Yau triangulated categories, Ann. Inst. Fourier (Grenoble) $\mathbf{5 8}$ (2008), 2221-2248. MR 2473635.

[22] - Cluster characters, II: A multiplication formula, Proc. Lond. Math. Soc. (3) 104 (2012), 57-78. MR 2876964. DOI 10.1112/plms/pdr027.

Thorsten Holm

Institut für Algebra, Zahlentheorie und Diskrete Mathematik

Fakultät für Mathematik und Physik

Leibniz Universität Hannover

30167 Hannover

Germany

holm@math. uni-hannover.de

http://www.iazd.uni-hannover.de/ tholm

Peter Jørgensen

School of Mathematics and Statistics

Newcastle University

Newcastle upon Tyne NE1 $7 R U$

United Kingdom

peter. jorgensen@ncl . ac.uk

http://www.staff.ncl.ac.uk/peter.jorgensen 